DOI: $10.17957 / \mathrm{IJAB} / 15.1778$

http://www.fspublishers.org

\title{
Transcriptome Analysis of Korla Fragrant Pear Reveals a Comprehensive Signaling Network in Response to Alternaria alternata Infection
}

\author{
Hui Ouyang ${ }^{\dagger}$, Tongrui Sun ${ }^{\dagger}$, Minrui Guo, Weida Zhang, Wenbo Guo, Ying Jiang, Shaobo Cheng ${ }^{*}$ and Guogang Chen \\ School of Food Science and Technology, Shihezi University, Shihezi 832000, P. R. China \\ "For correspondence: 1312303432@qq.com; cgg611@163.com \\ ${ }^{\dagger}$ Contributed equally to this work and are co-first authors \\ Received 09 December 2020; Accepted 08 February 2021; Published 10 May 2021
}

\begin{abstract}
Blackhead caused by Alternaria alternata is a fatal necrotrophic fungal that affects Korla fragrant pear. To date, little is known at the molecular level about the defense response of pear to blackhead disease and the pathogenic mechanism of $A$. alternata infection. To investigate the specific host-pathogen interaction between A. alternata and pear, we examined the accumulation of host-responsive mRNAs using RNA-seq technology. A total of 25,877 differentially expressed genes (DEGs) were identified. Further analysis revealed that the DEGs mainly participate in plant cell wall integrity, plant hormone pathways, plant-pathogen interactions and the defense response (transcription factors, defense-related proteins). Most of the DEGs involved in the plant hormone, PAMP-triggered immunity (PTI) and effector-triggered immunity (ETI) pathways, as well as defense-related proteins, were significantly up-regulated. In addition, DEGs encoding enzymes involved in cutin and wax synthesis and most transcription factors are significantly down-regulated. Based on these results, we speculate that these pathways play important roles in the response of pear to A. alternata. This study has presented new insights into the molecular mechanisms that regulate the response of pear fruits to A. alternata infection. (C) 2021 Friends Science Publishers
\end{abstract}

Keywords: Alternaria alternata; Blackhead disease; Defense response; Infection; Korla fragrant pear; RNA-seq

\section{Introduction}

Korla fragrant pear (Pyrus sinkiangensis $\mathrm{Yu}$ ) is a traditional high-quality fruit grown in Xinjiang province, China (Ma et al. 2019). It is popular with consumers because of the thin exocarp, crispy flesh, high juice and sugar content and pleasant rich fragrance (Chen et al. 2020). At present, Korla fragrant pear is exported to many countries around the world and has high commercial value on the international market due to its special flavour and nutritional qualities (Tian et al. 2014). Unfortunately, Korla fragrant pear is prone to diseases such as blackhead disease, scab, powdery mildew, brown spots, fire blight and other fungal diseases during long-distance transportation or long-term storage (Cheng et al. 2019). Among these diseases, blackhead disease mainly occurs during storage and its incidence rate can be as high as $10 \%$, making blackhead the main storage disease of Korla fragrant pears.

Blackhead disease caused by Alternaria alternata is a fatal necrotrophic fungal disease that affects Korla fragrant pear quality and production (Chen et al. 2019). At the early stage of fruit infection, the lesions first appear at the calyx end of the pear, the peel in the diseased area turns brown- black, the flesh appears as a light brown honeycomb and the uninfected pulp tissues look good but taste slightly bitter. As the disease development progresses, the surface of the fruit collapses and produces a sticky black juice-like substance. A distinct boundary appears between the lesion and the internal good flesh and a white mold layer grows on the peel that leads to a decline in pear fruit quality. At present, chemical fungicides are the main method used to inhibit fungal disease (Dalcero et al. 1996). However, long-term and large-scale use of chemical fungicides may lead to strong resistance in A. alternata and have an adverse effect on the environment (Ma and Michailides 2005). Therefore, an improved description of the host-pathogen interaction and the pear defense response against $A$. alternata infection will help provide a reference for understanding the causes of blackhead disease and biological control of the pathogen.

With the rapid development of molecular biology and associated bioinformatics tools, considerable progress has been made in understanding plant-pathogen interactions. RNA-seq technology is an important tool to explore complex biological processes (Marguerat and Bähler 2010). At present, RNA-seq technology has been frequently used to study the interaction between plants and pathogens in

To cite this paper: Ouyang H, T Sun, M Guo, W Zhang, W Guo, Y Jiang, S Cheng, G Chen (2021). Transcriptome analysis of korla fragrant pear reveals a comprehensive signaling network in response to Alternaria alternata infection. Intl J Agric Biol 25:1173-1186 
horticultural crops. For example, RNA-seq analysis have provided valuable information about changes in gene expression in host-pathogen interactions involving apple and A. alternata (Zhu et al. 2017), apple and powdery mildew fungi (Tian et al. 2019), Callery pear and A. alternata (Kan et al. 2017). In this study, RNA-seq was used to explore the transcriptomic profiles of Korla pears in response to fungal infection. Our objective is to explore the potential causes of susceptibility of pears to A. alternata infection at the molecular level, so as to provide support for the development of new storage and preservation technologies for pear fruits.

\section{Materials and Methods}

\section{Plant materials, A. alternata culture and inoculation procedures}

Five-year-old Korla fragrant pear plants were grown in a pear orchard located in Xinjiang province, China. This study cultured the A. alternata fungus on potato dextrose agar (PDA; $20 \mathrm{~g}$ dextrose, $200 \mathrm{~g}$ potato extract and $20 \mathrm{~g}$ agar in 1 $\mathrm{L}$ of water) medium at $28^{\circ} \mathrm{C}$ in the dark. After 5 days, conidia were collected in $\sim 20 \mathrm{~mL}$ of distilled sterile water per plate by swirling gently to detach the conidia. The conidial suspension was then centrifuged and the conidia were diluted until reaching the concentration of $1 \times 10^{5}$ conidia $/ \mathrm{mL}$. The surface of pears was disinfected with $75 \%$ alcohol and then rinsed in sterile distilled water for 3 times. After air drying, 3 holes were punched in the surface of the pears $($ diameter $=1 \mathrm{~mm}$; deep $=1 \mathrm{~mm})$ and $20 \mu \mathrm{L}$ of the conidial suspension was introduced in these holes using a pipette. The control group (T0) was inoculated with sterile water. There were four experimental pear groups, each consisting of 30 inoculated fruits. The fruits were incubated in sterile plastic chambers at $25^{\circ} \mathrm{C}$ under a $14 \mathrm{~h}$ light $/ 10 \mathrm{~h}$ dark cycle and fruit tissues around the wounded sites $(1 \mathrm{~cm}$ deep x $1 \mathrm{~cm}$ diameter)) were taken at 24, 72 and 96 hours as experimental groups T1, T3 and T5, respectively. After flash freezing in liquid nitrogen, the fruit samples were stored at $-80^{\circ} \mathrm{C}$ for later use during the RNA-seq experiments. Each stage involved three parallel fruit samples which were picked at the same time and represented three biological replicate samples, respectively.

\section{Total RNA extraction, library construction and sequencing}

The cetyltrimethyl ammonium bromide (CTAB)-based method was used to isolate total RNA and to improve the RNA isolation, column purification was performed using a RNAeasy Plant Mini kit produced by Qiagen (Germany). The three biological replicates at different stages of infection were combined to construct a complementary DNA (cDNA) library. Next, magnetic beads were used to purify the products and oligo (dT) magnetic beads were used to enrich mRNAs. Then short fragments of $\sim 200$ bases were prepared by mixing the mRNAs were with fragmentation buffer and they were used as templates to synthesize first-strand cDNA and random hexamer-primers. Second-strand cDNAs were synthesized using Buffer, dNTPs, RNase H and DNA polymerase I. The short twostranded cDNAs were subject to purification via a QiaQuick PCR extraction kit. After repair, the cDNA fragment ends and poly (A) tails were connected to the Illumina sequencing adapters. Fragments of different size ranges were separately recovered by agarose gel electrophoresis and fragments of the appropriate sizes were enriched by PCR amplification. The constructed libraries were subject to sequencing using an $\mathrm{n}$ Illumina $\mathrm{HiSeq}^{\mathrm{TM}}$ platform.

\section{RNA-seq data analysis}

We converted the raw image data obtained from the sequencing instrument into raw sequence reads and saved it in FASTQ file format. Raw data were used so that highquality clear reads for subsequent analysis can be obtained and they were trimmed to remove the sequencing adapters. Reads consisting only of adapters, those with $>10 \%$ Ns (unknown bases) and low-quality reads in which the percentage of bases with phred quality scores $\mathrm{Q} \leq 20$ was $>50 \%$ were removed from the data sets.

The relative gene expression level was calculated using Fragments Per Kilobase of transcript per Million mapped reads (FPKM). The criteria for identifying differentially expressed genes (DEGs) were a False Discovery Rate $(\mathrm{FDR}) \leq 0.05$ and the absolute value of $\mid$ fold-change $(\mathrm{FC}) \mid \geq 1$. Annotation of proteins encoded by the DEGs was performed by GO functional classification using the Blast2GO program. We then used WEGO software to analyze the significant functional enrichment of the DEGs. Finally, the GO terms that showed significant enrichment in the DEGs was founded using the hypergeometric test with $P \leq 0.05$ as the threshold. We also used the DEGs as queries to search the KEGG database to analyze pathway enrichment using the same criteria described above.

\section{qRT-PCR verification}

We selected 10 random DEGs for qRT-PCR to analyze and verify the RNA-seq data. Based on the sequence information of the selected DEGs, we designed genespecific primers for qRT-PCR using the Primer Express software. Total RNA samples $(1 \mu \mathrm{g})$ from pears were used for cDNA synthesis by reverse transcription using the FastQuant RT Kit (Beijing, China) as directed by the manufacturer. The qRT-PCR assays were used on an ABI StepOnePlus Real-Time PCR System (ABI, U.S.A.) and the reaction mixtures $(20 \mu \mathrm{L})$ contained $0.4 \mu \mathrm{L}$ of the forward and reverse PCR primers $(10 \mu \mathrm{M}), 10 \mu \mathrm{L}$ of qPCR Master Mix and $4 \mu \mathrm{L}$ template cDNA. The amplification program 
was $95^{\circ} \mathrm{C}$ for $90 \mathrm{~s}$ and then 40 cycles of $95^{\circ} \mathrm{C}$ for $5 \mathrm{~s}, 60^{\circ} \mathrm{C}$ for $15 \mathrm{~s}$ and $72^{\circ} \mathrm{C}$ for $20 \mathrm{~s}$. Internal control on normalization of gene expression was performed using the pear Actin gene (Actin2/7) and the $2^{-\triangle} \Delta^{\mathrm{Ct}}$ method was used to calculate the relative expression level of selected unigenes. Three independent biological replicates were selected for each sample and Table 1 shows all the primers used.

\section{Results}

\section{Microscopic analysis of Korla fragrant pears infected with $A$. alternata}

By observing the symptoms of A. alternata-infected pears at different time points, we found that a few spores and hyphae were newly produced on the surface of the peel at 1 day post infection (dpi) (Fig. 1A). At 3 dpi, the spotted brown lesions on the surface of the pears were stained with white mycelia and the early-stage symptoms of blackheads became apparent (Fig. 1B). After 5 dpi, noticeable lesions appeared in the inoculated sites and were covered with large areas of white hyphae accompanied by softening and depression of the peel and pulp tissue (Fig. 1C).

To further determine the optimal sampling time and observe the process of $A$. alternata infection of fragrant pears, scanning electron microscopy and lactophenol trypan blue staining were used to observe the process of spore germination and hyphal production and growth. Microscopic observation showed that the spores start to germinate at $1 \mathrm{~d}$ and formed embryo tubes. Three days after inoculation, the germ tubes were further extended and the hyphae showed obvious growth and adhered to the surface of the peel. On the fifth day following inoculation, the surface of the peel including the inoculation site was covered with white hyphae and the hyphae had invaded the pulp tissue, causing symptoms such as brown lesions, softening and surface depression (Fig. 1D-I).

\section{RNA-seq data and DEG profiles in response to $A$. alternata infection}

Changes in transcript levels in comparisons of the noninoculated control and the inoculated groups (T0 vs. T1, T0 vs. T3 and T0 vs. T5) were determined in an RNA-seq experiment. We obtained a total of 196.25 million raw 300 bp paired end sequencing reads. After filtering out the lowquality reads, 191.83 million clean reads remained. Of these, 91.35 million $(48.10 \%)$ were mapped to the pear genome reference sequence and 87.20 million $(44.09 \%)$ of the clean reads mapped uniquely to the pear genome reference sequence. Based on these mapped reads, the expression levels of 13,621 DEGs were calculated using the FPKM method.

Gene expression between the A. alternata infected groups (T1, T3 and T5) and the control group (T0) were compared. The results identified 5,877, 7,970 and 11,485
DEGs at 1, 3 and $5 \mathrm{~d}$, respectively (Fig. 2A). Among the DEGs, there was no significant difference in the number of up-regulated genes, but the number of down-regulated genes increased gradually with the severity of infection. For example, there were 3,499, 3,580 and 3,503 up-regulated genes at 1, 3 and $5 \mathrm{~d}$, but 2,378, 4,390 and 7,987 genes were down-regulated at these three time points (Fig. 2B). Most of the DEGs were detected at $5 \mathrm{dpi}$, which suggests that this is a critical stage in the host response to A. alternata infection in pear fruits.

In addition, we visualized the number of DEGs from the different comparisons in a Venn diagram, which clearly shows that both unique DEGs and shared DEGs are present in the different groups (Fig. 2C). Among them, 3,958 DEGs were shared among the three pairwise comparisons and the T0 vs. T1 comparison shared 4,494 and 4,308 DEGs with the T0 vs. T3 and T0 vs. T5 comparisons, respectively. Moreover, there were 7,415 DEGs shared between the T0 vs. T3 and T0 vs. T5 comparisons, which is significantly higher than in the above two comparisons.

\section{Functional annotation of the DEGs}

To explore the function of the DEGs, a GO analysis was carried out by mapping them to three major GO categories, biological process, molecular function and cellular component when the corrected $P$-value was $<0.05$. In the biological process category, the GO terms "metabolic process" (2,577 DEGs), "cellular process" (2,184 DEGs) and "single-organism process" (1,699 DEGs) were the most enriched groups. In the molecular function category, "catalytic activity" (2,544 DEGs) and "binding" (1,690 DEGs) were remarkably enriched and we found that these two terms were highly involved in the process of plant hormone signal transduction. For cell component, the significantly enriched terms were "cell” (983 DEGs), "cell part" (983 DEGs), "macromolecular complex" (305 DEGs), "membrane" (784 DEGs), "membrane part" (616 DEGs), "organelle" (654 DEGs) and "organelle parts" (336 DEGs) (Fig. 3).

To identify pathways that play key roles in plantpathogen interactions, 8,512 DEGs were further assigned to 45 different categories by KEGG pathway analysis. The results showed that several secondary metabolite pathways involved in defense, such as those involved in synthesis of terpenoid derivatives and flavonoids, were enriched. These pathways included "tropane, piperidine and pyridine alkaloid biosynthesis", "ubiquinone and other terpenoid-quinone biosynthesis", "monoterpenoid biosynthesis" and "terpenoid backbone biosynthesis" (Table 2). Moreover, the pathways for defense signaling transduction and plant pathogen recognition, which are related to "plant hormone signal transduction", "cutin, suberine and wax biosynthesis" and "plant-pathogen interaction" were also enriched. However, the timing of gene induction was different. For instance, the pathways "plant hormone signal transduction" and "cutin, 
Table 1: Primers used for qPCR

\begin{tabular}{llll}
\hline Gene ID & Gene Name & Forward primer $\left(5^{\prime}-3^{\prime}\right)$ & Reverse primer $\left(5^{\prime}-3^{\prime}\right)$ \\
\hline- & Actin2/7 & CTCCCAGGGCTGTGTTTCCTA & CTCCATGTCATCCCAGTTGCT \\
LOC103936633 & EMB1144 & CTGTGCCGATGGTGGAAG & AGTTGCTGCCTCCGCT \\
LOC103939953 & PLD1 & CCCCCTTCCATTCACTTTTCAG & ACCACCTTGCTTTCTCCACC \\
LOC103943393 & IMP1 & ATTCGCTTGCTCAGTTCCTCT & CCTTATCAGTTTCCGTGACCAG \\
LOC103948139 & FBA3 & GACGAACTCCTCCTAACTGCC & CTTCCCATCGGTTGTAGACTG \\
LOC103951141 & ADH & CACCACCACAGGCAAATGAAG & TGTCACGCCCTCACCAATAC \\
LOC103951803 & At5g47720 & ATGGGTGGTTTTCTGGGTGC & CTGTATGCTCTGTGCTGCGA \\
LOC103957712 & Cht5 & AACAGGTCAAGTTTCGGTGG & CAAGAAAAGATTGCCGTGTGTAG \\
LOC103958948 & FQR1 & TTGCTGAGGCTGATGGGATA & GCTGTCAAGGGGGTAGTCTC \\
LOC103965128 & PDC4 & GGGACGCACAGGATTCTTCA & CCTGATAGCAAGTCACGGTCTG \\
LOC103966218 & DPE2 & GTCTGCTACTGAGCCCTGTC & ACATTTGAAGCCCTTTGGAAC \\
\hline
\end{tabular}

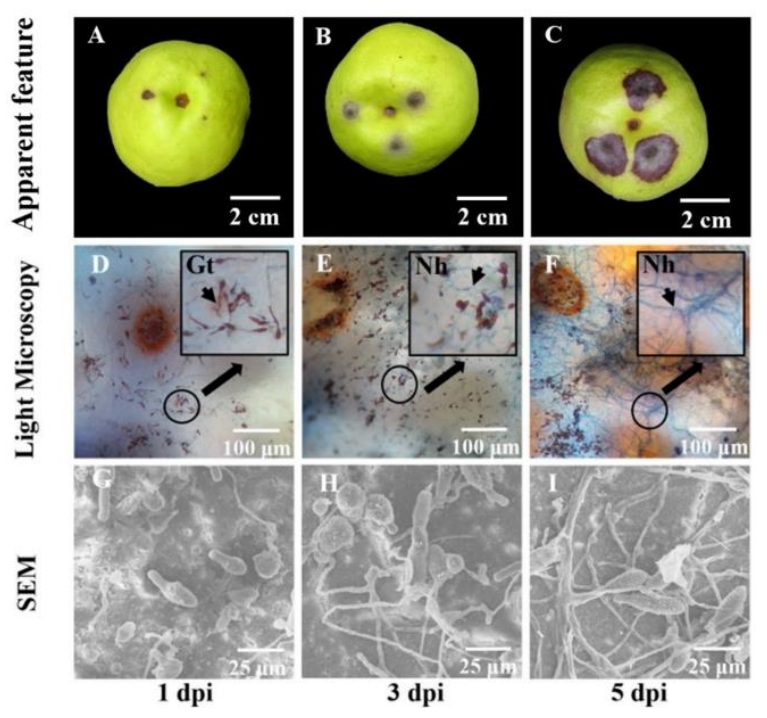

Fig. 1: Symptoms of $A$. alternata infection of Korla fragrant pear. Note: (A-C) Changes in symptoms of Korla fragrant pear after $A$. alternata infection; (D-F) Lactol trypan blue staining of pear epidermis inoculated with A. alternate; (D) The spores gradually germinate and form germ tubes; (E) Germ tube grows and produces a little mycelium; (F) White mycelium further invaded and expanded, producing obvious lesions at the inoculation site. GT, germ tube; Nh, New hypha; (G-I) Scanning Electron Microscopy observation of infection of Korla fragrant pear by A. alternata

suberine and wax biosynthesis" were induced earlier on the first day, while the "plant-pathogen interaction" pathway was induced later on the 3rd and 5th days.

\section{DEGs involved in plant cell wall integrity}

The plant cell wall is an important physical barrier against pathogen infection and is the main composition of the monitoring system in the plant innate immune system. There are many genes involved in plant cell wall biosynthesis, including HTH (Hothead) (Kurdyukov et al. 2006), WSD (Wax-ester synthase/ diacylglycerol oacyltransferase) (Li et al. 2008), GPAT (Glycerol-3phosphate acyltransferase) (Gidda et al. 2009), CER (Eceriferum) (Aarts et al. 1995), PME (Pectin methylesterase), (Bethke et al. 2014) and XTH (xyloglucan endotransglycosylase/hydrolase) (Rose et al. 2002). In this study, the RNA-seq data showed that expression of two GPAT6 (LOC103931179; LOC103949465), one HTHI (LOC103929758), two CERI (LOC103932050;
LOC103942391), three WSD1 (LOC103928845; LOC103941920; LOC103961411) and 13 XTH genes was down-regulated during the infection process (Table 2). In addition, we found that expression of 10 PME genes showed an upward trend after plants were infected with $A$. alternata (Fig. 4).

\section{DEGs involved in plant hormone signaling pathways}

Plant hormones, including salicylic acid (SA), jasmonic acid (JA), ethylene (ET), abscisic acid (ABA) and brassionosteroids (BRs), are critical factors involved in the plant disease defense response (Tian et al. 2019). To identify DEGs associated with hormonal responses in pears infected with A. alternata, we analyzed the hormone signal transduction pathways. In this study, three DEGs involved in SA signaling ( $P R-1$, Pathogenesis-related proteins) and six DEGs in JA signaling (JAZ, Jasmonate zim domain) were up-regulated (Fig. 5A- B); three DEGs involved in ET signaling encoding ERF1/2 (Ethylene response factor 1/2) 

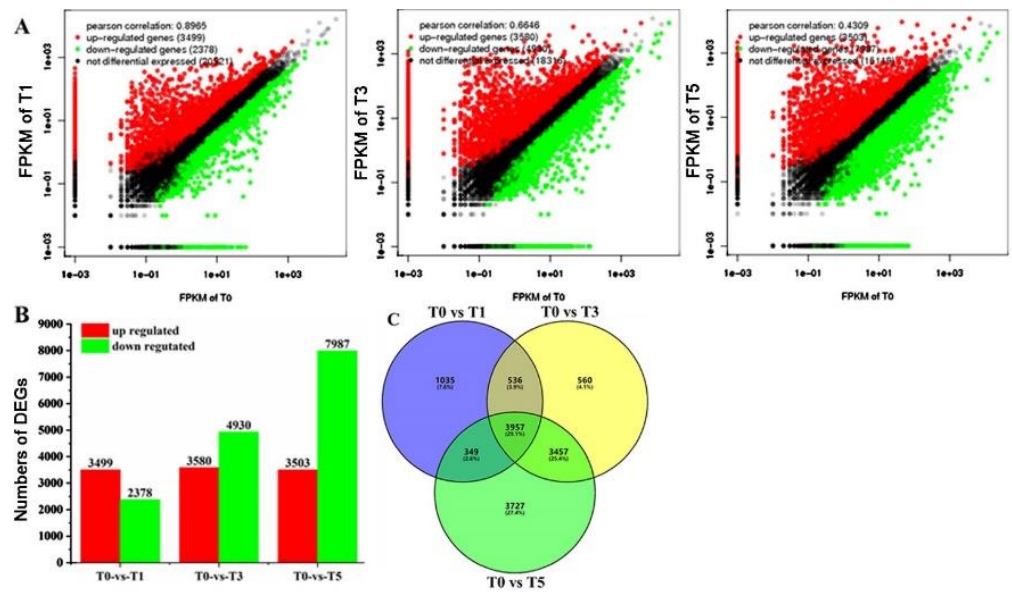

Fig. 2: DEGs between samples. (A) Scattered plot of differential expression. (B) Numbers of DEGs compared between two samples (T0 vs. T 1 dpi, T0 vs. T3 dpi, T0 vs. T5 dpi and with T0 dpi as the control). Red shows up-regulated, green shows down-regulated. (C) Venn diagram analysis of the DEGs in Korla fragrant pear after A. alternata infection

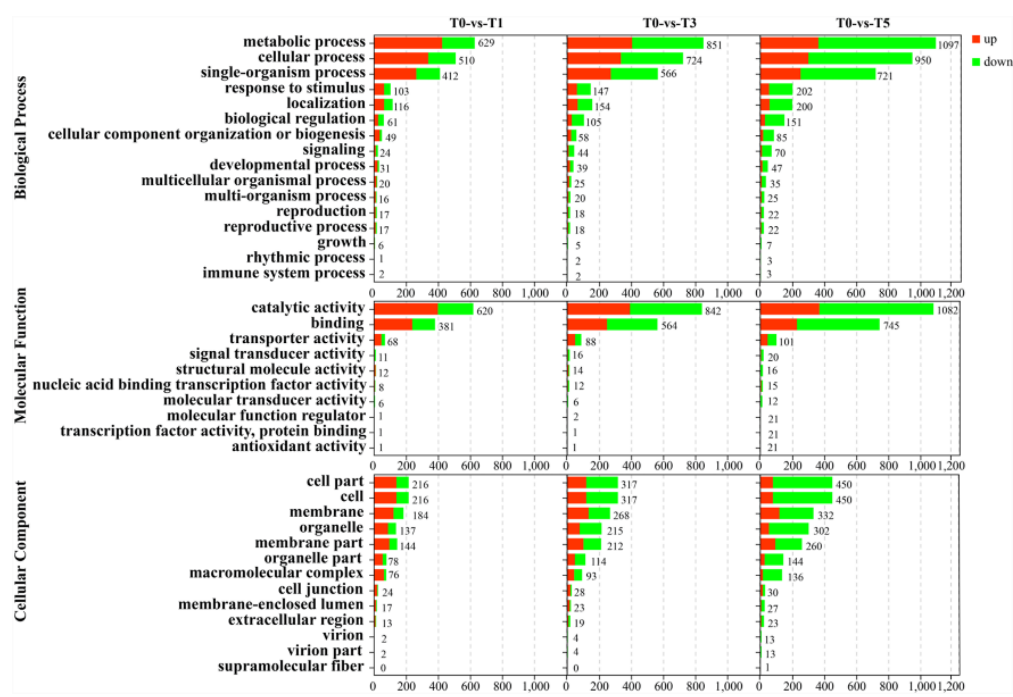

Fig. 3: GO categories of DEGs in Korla fragrant pear in response to A. alternata infection (red for up-regulated, green for down-regulated)

were up-regulated in the three stages (Fig. 5C); In addition, the genes involved in the ABA signal perception and transduction pathway, for example PYR/PYL (pyrabactin resistance1/PYR1-like) were up-regulated (Fig. 5D); DEGs involved in the BR-response, such as BRII (BRI-associated receptor kinase 1), $B S K$ (brassionosteroid insensive) and TCH4 (Xyloglucan endotransglucosylase, also known as Touch 4 ) that showed the same expression pattern were significantly upregulated at 5 dpi (Fig. 5E).

\section{DEGs involved in plant-pathogen interactions}

To identify DEGs associated with plant-pathogen interactions in the pears infected with A. alternata, we analyzed the plant-pathogen interaction pathways. In the present study, three DEGs encoding CDPK (calciumdependent protein kinases) and two DEGs encoding Rbohs (respiratory burst oxidase homologue) were up-regulated in the three stages, inducing a hypersensitive response and cell wall reinforcement. In addition, $16 \mathrm{CNGC}$ (cyclic nucleotide-gated ion channel) and 31 CaM/CML (calmodulin/cam-like) genes were regulated and among them, four DEGs encoding CNGC and six DEGs encoding $\mathrm{CaM} / \mathrm{CML}$ were strongly up-regulated at $3 \mathrm{dpi}$ and $5 \mathrm{dpi}$. Furthermore, four DEGs encoding RPM1 (RPM1interacting protein) were also up-regulated at 3 and $5 \mathrm{dpi}$ (Fig. 6). In addition two transcription factor genes, WRKY33 and WRKY29, showed changes in expression; these genes encode proteins that participate in the MAPK (mitogenactivated protein kinase) signaling pathway and induce plant disease resistance.

\section{DEGs encoding transcription factors (TFs)}

TFs are important regulatory proteins that can regulate gene transcription by binding to specific sequence motifs in the 
Ouyang et al. / Intl J Agric Biol, Vol 25, No 6, 2021
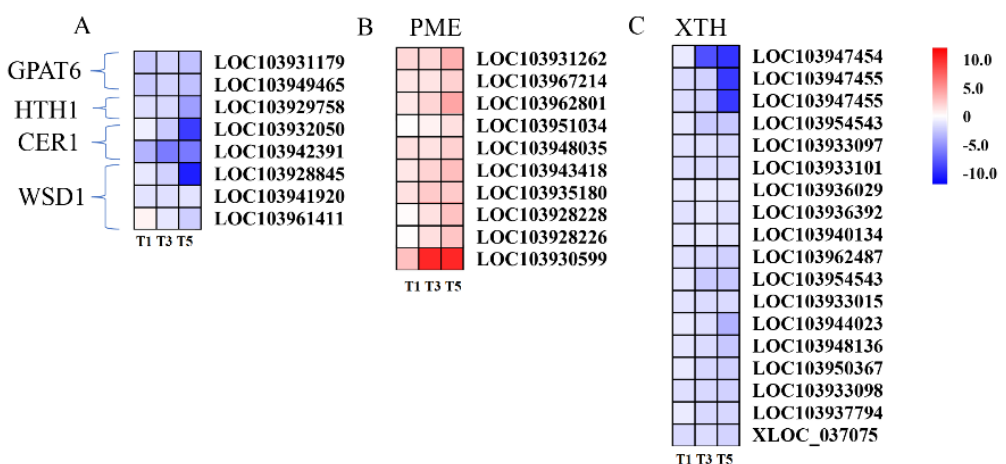

Fig. 4: Heatmaps of DEGs involved in plant cell wall integrity. The $\log _{2}{ }^{\mid \text {Foldchange| }}$ was colored using OriginPro 2020 (red for up-regulated, green for down-regulated), each horizontal row represents a DEG with its gene ID and the vertical columns represent 1, 3 and 5 dpi from left to right. (A) DEGs related to stratum corneum; (B) Genes related with PME; (C) Genes related with XTH

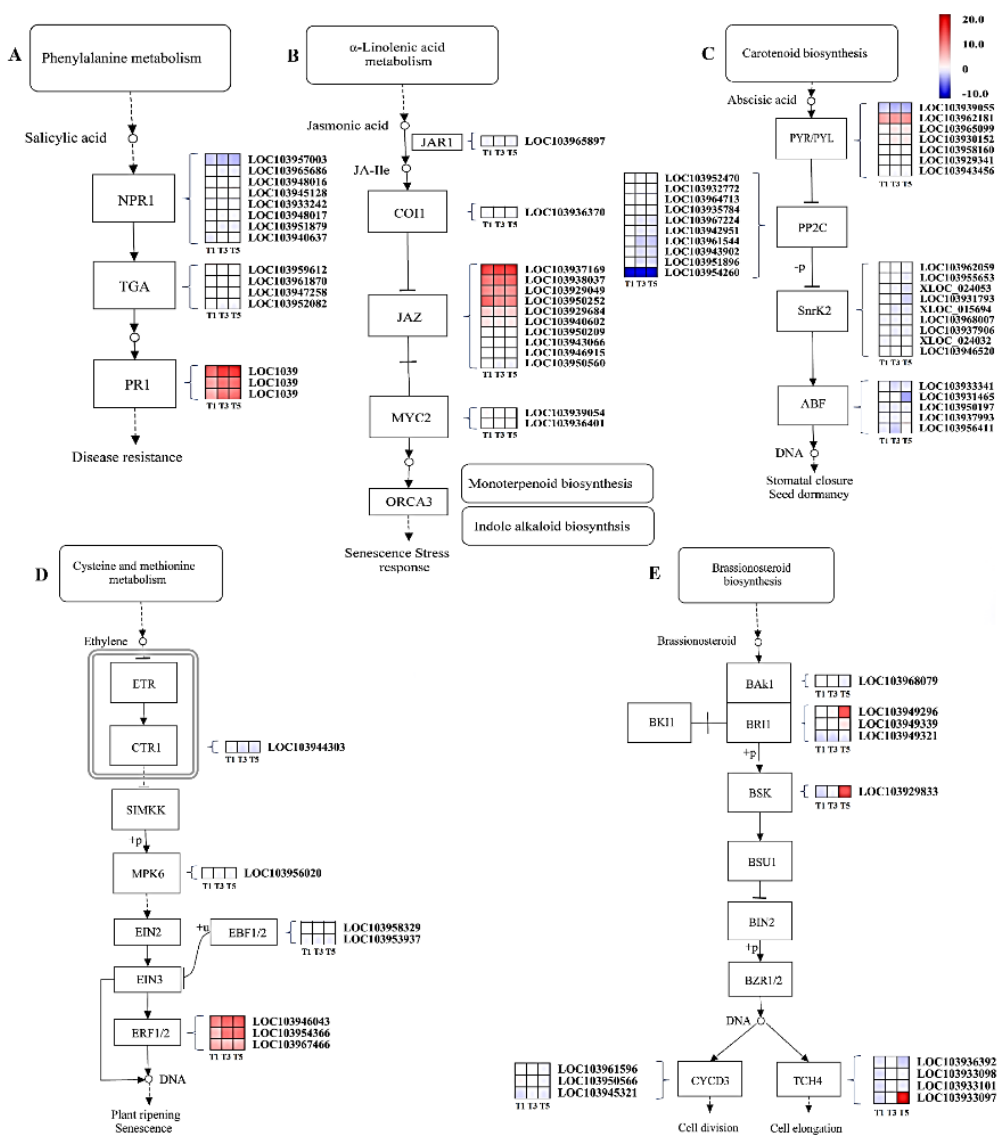

Fig. 5: Heatmaps of DEGs Involved in Plant Hormone Signaling Pathway. The $\log _{2}{ }^{\mid \text {Foldchange| }}$ was colored using OriginPro 2020 (red for up-regulated, green for down-regulated), each horizontal row represents a DEG with its gene ID and the vertical columns represent 1, 3 and 5 dpi from left to right. (A) DEGs related to SA; (B) Genes related with JA; (C) Genes related with ET; (D) Genes related with ABA; (E) Genes related with BRs

promoter regions of downstream target genes (Vidhyasekaran 2016). In plants, WRKY, MYB, ERF, Hsfs, ZIP and NAC are all important transcriptional regulators of plant defense responses (Pandey and Somssich 2009; Dezar et al. 2011; Pajerowska-Mukhtar et al. 2012). In this study, several TF-encoding genes belonging to different families were either up- or down-regulated, including 54 WRKY genes, $29 M Y B$ genes, 12 ERF genes, $17 b H L H$ genes, nine $A R F$ genes, six $H s f$ genes, two ZIP genes and two $D O F$ genes (Fig. 7). Interestingly, more DEGs were down-regulated than up-regulated in the MYB, ERF, ARF, Hsfs, ZIP and bHLH families. The expression of related TF genes in pear fruits after A. alternata infection is shown in Fig. 7. 
Table 2: Significantly enriched KEGG pathway of DEGs in response to A. alternata

\begin{tabular}{|c|c|c|c|c|}
\hline \multirow[t]{2}{*}{ Pathway } & \multicolumn{3}{|c|}{ Number of DEGs at each time point } & \multirow[t]{2}{*}{ Pathway ID } \\
\hline & T0 vs. T1 & T0 vs. T3 & T0 vs. T5 & \\
\hline Biosynthesis of secondary metabolites & 396 & 556 & 693 & ko01110 \\
\hline Terpenoid backbone biosynthesis & 22 & 35 & 46 & ko00900 \\
\hline Taurine and hypotaurine metabolism & 13 & & 17 & ko00430 \\
\hline Biosynthesis of antibiotics & 164 & 234 & 294 & ko01130 \\
\hline Glycolysis / Gluconeogenesis & 45 & 65 & 83 & ko00010 \\
\hline Circadian rhythm - plant & & 28 & 38 & ko04712 \\
\hline Photosynthesis & & & 37 & ko00195 \\
\hline Metabolic pathways & 576 & 842 & 1080 & ko01100 \\
\hline Carotenoid biosynthesis & 17 & 23 & 31 & ko00906 \\
\hline Pyruvate metabolism & & 46 & 63 & ko00620 \\
\hline Porphyrin and chlorophyll metabolism & & & 36 & ko00860 \\
\hline alpha-Linolenic acid metabolism & 35 & 40 & 43 & ko00592 \\
\hline Regulation of autophagy & & 23 & 31 & ko04140 \\
\hline Sulfur metabolism & & 25 & 30 & ko00920 \\
\hline Folate biosynthesis & & 10 & 14 & ko00790 \\
\hline Phenylalanine, tyrosine and tryptophan biosynthesis & 27 & 29 & 37 & ko00400 \\
\hline Vitamin B6 metabolism & 10 & 10 & 14 & ko00750 \\
\hline Sesquiterpenoid and triterpenoid biosynthesis & & 18 & 19 & ko00909 \\
\hline Ubiquinone and other terpenoid-quinone biosynthesis & & & 27 & ko00130 \\
\hline Phenylalanine metabolism & & 22 & 28 & ko00360 \\
\hline Pentose phosphate pathway & & 30 & 35 & ko00030 \\
\hline Arginine and proline metabolism & 23 & 30 & 36 & ko00330 \\
\hline Tropane, piperidine and pyridine alkaloid biosynthesis & 16 & 19 & 20 & ko00960 \\
\hline Plant-pathogen interaction & & 97 & 114 & ko04626 \\
\hline Steroid biosynthesis & & 22 & 27 & ko00100 \\
\hline Tyrosine metabolism & 22 & 25 & 27 & ko00350 \\
\hline Selenocompound metabolism & & 10 & 12 & ko00450 \\
\hline Monobactam biosynthesis & 8 & 7 & 8 & ko00261 \\
\hline Flavonoid biosynthesis & 18 & 30 & & ko00941 \\
\hline Ribosome & 152 & 161 & & ko03010 \\
\hline Monoterpenoid biosynthesis & 8 & 10 & & ko00902 \\
\hline Cysteine and methionine metabolism & 41 & 54 & & ko00270 \\
\hline Carbon fixation in photosynthetic organisms & 30 & 39 & & ko00710 \\
\hline Pantothenate and CoA biosynthesis & & 21 & & ko00770 \\
\hline Carbon metabolism & & 118 & & ko01200 \\
\hline beta-Alanine metabolism & 21 & 23 & & ko00410 \\
\hline Glutathione metabolism & & 44 & & ko00480 \\
\hline Fatty acid degradation & 20 & & & ko00071 \\
\hline Butanoate metabolism & 11 & & & ko00650 \\
\hline Cutin, suberine and wax biosynthesis & 15 & & & ko00073 \\
\hline Plant hormone signal transduction & 87 & & & ko04075 \\
\hline
\end{tabular}

\section{DEGs encoding defense-related proteins}

Exposure to abiotic and biotic stresses triggers the expression of various defense-related proteins with antibacterial activity, which leads to other defenserelated responses such as cell death, the HR and cell wall rigidification in plants. In our results, a number of DEGs encoding defense-related proteins belonging to different families were up-regulated at three detection time points; these included three $P R-1$ genes, $10 C H T$ (chitinase) genes, six TLP (thaumatin-like protein) genes and seven POD (peroxidase) genes (Fig. 8). In contrast to the above four TF families, genes encoding HSP family proteins were significantly down-regulated (Fig. 8).

\section{qRT-PCR verification}

In order to verify the RNA-seq results, we randomly selected 10 DEGs from two time points (T0 and T3) of pear fruit infection for qRT-PCR analysis. The genes were shown to be either up- or down-regulated in the T0 vs. T3 comparison and our analysis showed that the qRT-PCR and RNA-seq data expression results were consistent and showed a significant positive correlation, verifying the accuracy and reliability of the RNA-seq data (Fig. 9).

\section{Discussion}

The Korla fragrant pear is a type of fruit that has both a high nutritional value and a high commercial value. However, 


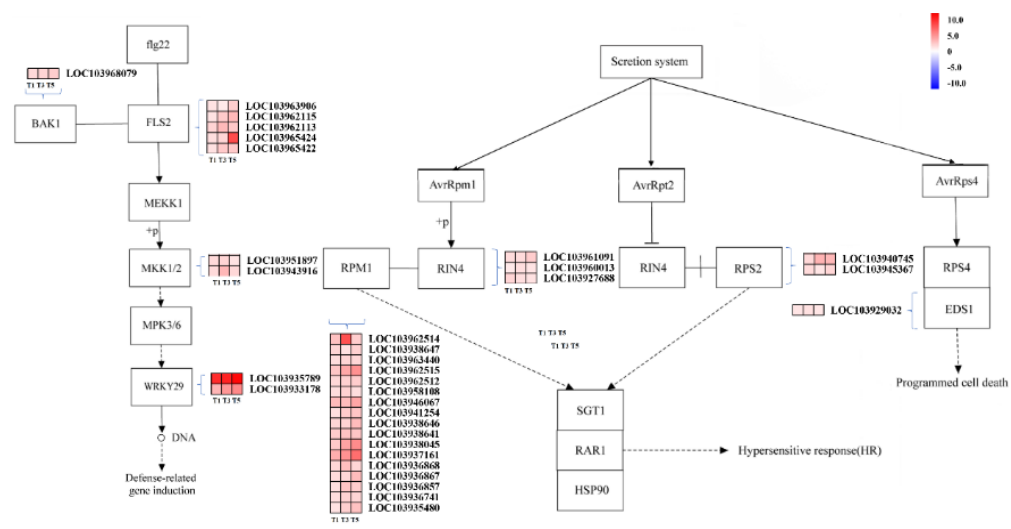

Fig. 6: Plant-Pathogen Interaction Pathway. The red for up-regulated, green for down-regulated (red for up-regulated, green for downregulated), each horizontal row represents a DEG with its gene ID and the vertical columns represent 1, 3 and 5 dpi from left to right. (A) DEGs related to PTI; (B) DEGs related to ETI

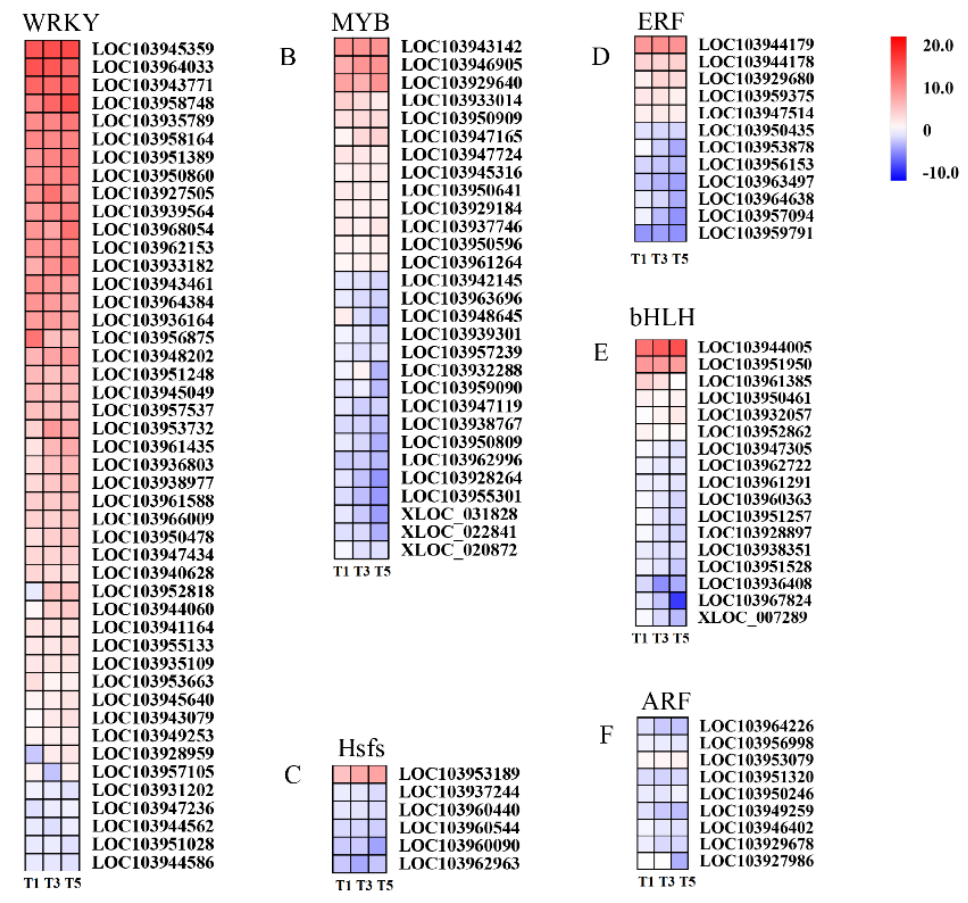

Fig. 7: Heatmaps of DEGs Involved in Transcription Factors. The $\log _{2}{ }^{\mid \text {Foldchange| }}$ was colored using OriginPro 2020 (red for up-regulated, green for down-regulated), each horizontal row represents a DEG with its gene ID and the vertical columns represent 1, 3 and 5 dpi from left to right. (A) DEGs related to WRKY; (B) Genes related with MYB; (C) Genes related with Hsfs; (D) Genes related with ERF; (E) Genes related with bHLH. (F) Genes related with ARF

during storage, fragrant pears are readily infected by $A$. alternata, which leads to great losses. Therefore, we studied the transcriptome changes in fragrant pear in response to $A$. alternata infection in order to explore the potential reasons for the susceptibility of pears to blackhead disease at the molecular level and to provide support for the development of new storage technology for pear fruits. At present, the reference genome of Chinese white pear maintained by NCBI has a total of 42,194 genes (Wu et al. 2013). In this study, 25,877 (61.33\%) genes were compared in all of the sample groups. The results of the enrichment analysis showed that the DEGs are mainly involved in metabolic pathways related to resistance, such as plant cell wall metabolic pathways, plant hormone signaling pathways, plant-pathogen interaction pathways and transcription factor regulation pathways, among others. Compared with other fruits, Korla fragrant pear has a number of unique characteristics. There is a dense wax layer on the surface of pear, which is very effective at maintaining fruit quality and controlling pathogenic microorganisms. The wax layer can further assist the plant cell wall and protect plant cells from microbial infection (Bellincampi et al. 2014). Many genes are involved in the biosynthesis of the plant cell wall and epidermal wax layer, including $H T H, W S D, G P A T, C E R$, 


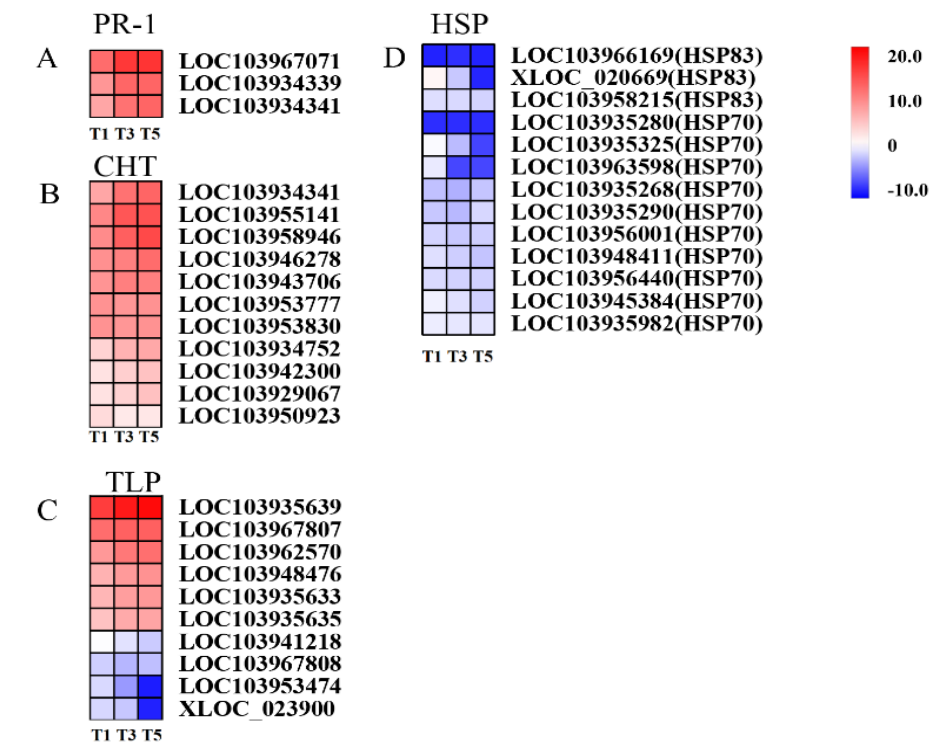

Fig. 8: Heatmaps of DEGs Involved in Defense-related proteins. The $\log 2^{\mid \text {Foldchange| }}$ was colored using OriginPro 2020 (red for up-regulated, green for down-regulated), each horizontal row represents a DEG with its gene ID and the vertical columns represent 1, 3 and 5 dpi from left to right. (A) DEGs related to PR-1; (B) Genes related with chitinase; (C) Genes related with TLP; (D) Genes related with HSP

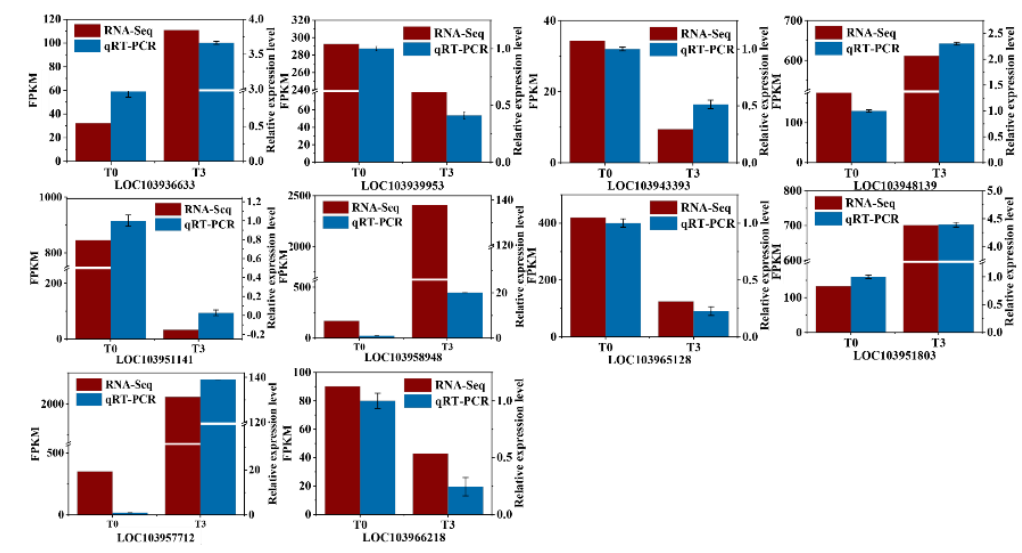

Fig. 9: Validation of RNA-seq data by qRT-PCR. 10 DEGs were selected for validation and they showed a similar tendency with RNASeq. Left vertical coordinate is RPKM of RNA-Seq; right vertical coordinate is relative expression level of qRT-PCR

$P M E$ and $X T H$. Our results show that the homologs of these genes in pear, GPAT6, HTH1, CERl and WSDl all showed down-regulated expression during the infection process. Previous studies have shown that the down-regulation of HTH1 and GPAT6 may have adverse effects on the formation of the stratum corneum (Ya et al. 2017), while the down-regulation of CERI and WSDI could affect the synthesis of the cuticular wax layer (Li et al. 2008). Therefore, the down-regulation of these genes in this study implies that the biosynthesis of the cuticle and wax is impaired in infected pears making it easier for A. alternata to penetrate, which may be the main reason why pears are more susceptible to pathogen infection. In addition, we found that $10 P M E$ genes showed an upward expression trend after plants were infected with A. alternata (Fig. 4). It is well known that PME can catalyze the demethylesterification of its pectin substrate. Therefore, the up-regulated expression of $P M E$ genes in this study may indicate that the degradation of plant cell wall components is accelerated.

Plant hormones are a general class of signaling molecules that play key regulatory roles in plant growth, development and defense responses. The complex interactions between different plant hormones via signaling pathways, called hormone crosstalk, can lead to changes in plant-specific metabolic pathways (Robert-Seilaniantz et al. 2011). The plant hormones usually involved in crosstalk are SA, JA, ET, ABA and BRs, which activate the corresponding defense reactions by regulating specific physiological responses, thereby preventing and resisting infection by pathogenic microorganisms (Bari and Jones 2009). In this study, genes involved in the SA $(P R-1)$, JA $(J A Z, M Y C 2)$, ABA $(P Y R / P Y L)$, ET $(E R F 1 / 2)$ and BRs (BRII, BSK, TCH4) signaling pathways were up-regulated 


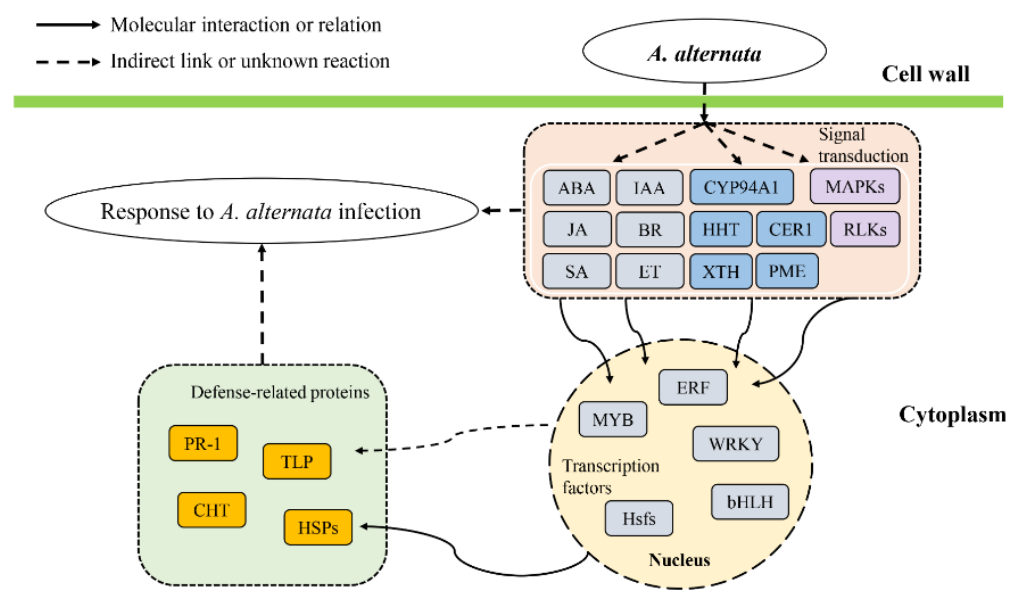

Fig. 10: Molecular network underlying the defense response to A. alternata in pear

when pears were infected with A. alternata. Some studies have shown that overexpression of $P R-1$ may play a positive role in enhancing plant immunity to pathogens (Tian et al. 2019). We found that several DEGs associated with SA ( $P R$ 1) were significantly upregulated in response to A. alternata infection, suggesting that SA might participate in regulating the response to A. alternata in pears. Previous studies have shown that JA and ET are mainly involved in the defense response against necrotrophs (Zhu et al. 2017). In this experiment, the genes involved in JA (JAZ, MYC2) and ET $(E R F 1 / 2)$ signaling were up-regulated when the pears were infected by $A$. alternata, which is consistent with previous studies. ABA is an important regulator of the interaction between plants and pathogenic microorganisms (Laurens et al. 2017). Many studies have shown that ABA often interferes with defense signaling pathways such as the SA/JA/ET pathway, thus negatively regulating plant resistance (Zhu et al. 2017). In this study, the genes involved in ABA signaling pathways, for example $P P 2 C, S n r K 2$ and $A B F$ were down-regulated while $P Y R / P Y L$ were upregulated in response to A. alternata infection, which revealed that the ABA signaling pathway was significantly inhibited after infection by $A$. alternata. This result is consistent with the response of apple leaves to A. alternata infection (Zhu et al. 2017). Finally, BRs play a complex and positive role in plant innate immunity (Tian et al. 2019). In this study, several DEGs associated with BR signaling were significantly upregulated in response to $A$. alternata infection, suggesting that BRs might participate in regulating the response to A. alternata infection in pear fruits.

In the process of resisting pathogen infection, plants mainly use two defense mechanisms. On the one hand, they trigger pathogen triggered immunity (PTI) by recognizing a broad range of pathogens with conserved molecular pattern on their surface and on the other hand, specific $\mathrm{R}$ genes that contain nucleotide binding site (NBS) and leucine-rice repeat (LRR) domains recognized specific pathogen proteins to trigger effector-triggered immunity (ETI) (Sun et al. 2013). In this study, we detected several genes involved in PTI and ETI that showed differential expression in response to A. alternata infection. BAK1 is one of the best studied receptor-like protein kinases (RLKs). A previous study has shown that flg22 induces BAK1 as a co-receptor and initiates immune signaling during the heterodimerization of FLS2 and BAK1 (Sun et al. 2013). In our research, the genes encoding BAK1 and FLS2 were upregulated after inoculation, suggesting that BAK1 and FLS2 may promote the immune response in pear and lead to resistance to $A$. alternata. MAPK cascades play an important role as signaling modules of a high conservation level in the response to abiotic and biotic stress and activate downstream defense-related genes (Colcombet and Hirt 2008). The MEKK1-MKK1/2-MPK4 signaling cascade in Arabidopsis thaliana affects both plant defense responses and the acquisition of basal resistance (Su et al. 2013). Our RNA-seq data revealed that the genes encoding the downstream targets of the MAPK cascades were upregulated, which may be help pear activate the innate immune system in response to pathogen infection to produce a related immune response. Pathogens usually secrete pathogen effectors to inhibit FLS2 recognition of flg22, thus enhancing the colonization and proliferation of pathogens by overcoming PTI (Crabill et al. 2010). At this time, in order to resist pathogen infection, plants will further induce ETI to trigger the HR response by recognizing viral effectors through specific disease-resistance proteins (Guo et al. 2009). In the present study, the genes encoding RIN4, RPM1, RPS2 and EDS1 were all up-regulated. Previous studies have reported that the effectors AvrRpm1 and AvrB secreted by Agrobacterium tumefaciens during infection of Nicotiana benthamiana plants can phosphorylate the RIN4 protein to relieve its negative regulation of the disease resistance (R) protein RPM1, which limits the occurrence of disease. AvrRpt2 abolishes the inhibition of RPS2 by RIN4 by removing the physical connection between RIN4 and RPS2, ultimately triggering an $\mathrm{R}$ protein-mediated $\mathrm{HR}$ 
response (Axtell and Staskawicz 2003). Therefore, the upregulation of genes encoding RPM1 and RPS2 may help to inhibit pathogen infection and activate the ETI immune system. EDS1 is a positive regulator of ETI and the upregulated expression of EDS1-encoding genes also activates programmed cell death (Bhattacharjee et al. 2011). Based on these results, we can infer that PTI and ETI play roles in resisting pathogen infection during the infection and colonization of pears by A. alternata.

Transcription factors (TF) are widely involved in plant responses to biotic stresses and regulate the expression of defense-related genes at the transcriptional level (Sun et al. 2013). In our RNA-seq data, WRKY TF family genes comprised the largest group and most of the WRKY genes were up-regulated. Previous studies have shown that WRKY family genes act as positive regulators in both the Arabidopsis response to Pectobacterium carotovorum ssp. carotovorum infection and in the response to A. alternata infection in apple leaves (Zhu et al. 2017). These results indicate that up-regulated expression of $W R K Y$ family genes in pear may play an important role in the response to $A$. alternata infection.

MYB family genes are mainly involved in various functions such as anthocyanin biosynthesis, morphogenesis and abiotic stress responses, among others (Wang et al. 2004). Zhang et al. (2020) found that MYB family genes in wheat can mediate host resistance to the fungal pathogen Bipolaris sorokiniana by regulating the SA signaling pathway and defense-related genes. Zhu et al. (2017) reported that $M Y B$ family genes might play a regulatory role in the responses of the 'Starking Delicious' pear cultivar to A. alternata attack. A total of 29 pear $M Y B$ genes were found to be either up- or down- regulated in response to $A$. alternata infection in this experiment (Fig. 7), which suggests that these genes might also play a regulatory role in the response of Korla pear fruits to A. alternata infection. However, further research is needed to confirm this hypothesis.

Heat shock transcription factors (Hsfs) participate in the response to biotic and abiotic stresses by regulating the expression of heat shock-related genes (Yu et al. 2019). A total of six pear Hsf genes were either up- or downregulated by $A$. alternata infection in this experiment (Fig. 7). Interestingly, we found that one of the down-regulated DEGs, LOC103937244, encodes a protein that is highly similar to AtHsfB2b from Arabidopsis. Kumar et al. (2009) reported that knockout of $A t H s f B 2 b$ in Arabidopsis can significantly improve resistance to the necrotrophic fungal pathogen A. brassicicola. We therefore speculate that the down-regulation of this gene in pear can improve disease resistance. The other four Hsf genes (LOC103962963, LOC103960544, LOC103960440, LOC103960090) also showed down-regulated expression, suggesting that the defense signal transduction pathway mediated by Hsf TFs may be compromised in Korla fragrant pear, leading to $A$. alternata infection.
Ethylene-responsive element binding factors (ERFs) are one family of TFs that are found only in plants (Cao et al. 2018). Yang et al. (2005) reported that the ERF4 and ERF12 genes in Arabidopsis encode transcriptional repressors that can modulate ethylene and abscisic acid responses. In this study, the genes encoding ERF4 (LOC103944178), ERF12 (LOC103944179) and ERF17 (LOC103944180) were significantly up-regulated (Fig. 7). Up-regulation of these genes negatively regulates ethylene and abscisic acid reactions, which may be related to the down-regulation of most genes in the abscisic acid and ethylene pathways (Fig. 5).

In addition, our results show that some other transcription factor family genes ( $b H L H$ and $A R F)$ were either up- or down- regulated by A. alternata infection (Fig. 7). However, various studies have shown that $b H L H$ and $A R F$ TFs play key roles in plant growth, development and stress tolerance, but are not stronly correlated with disease resistance (Zhang et al. 2020). Therefore, we will not give more details on these TF families here.

The up-regulation of defense-related protein genes has been found in a variety of plants and it is an inducible part of the plant's self-defense mechanisms (Jwa et al. 2006). Expression of genes that encode members of the PR-1 protein family was detected in pears and most of them were found to be regulated in A. alternata-infected fruits. The PR1 protein was first detected in tobacco plants infected with Tobacco mosaic virus and is the main PR protein induced by pathogen infection and SA (Loon and Kammen et al. 1970). PR-1 homologues are also found in wheat, corn and tomato plants infected by pathogens and elevated PR-1 protein levels in host plants also increase resistance to pathogens (Niderman et al. 1995). The genes encoding PR-1 proteins were found to be up-regulated in our experimental results and this may play a positive role in disease control. It is worth noting that up-regulation of the PR-1 protein gene is induced by the up-regulation of SA signaling pathway genes, suggesting that SA can increase resistance to necrotrophic fungi infection by inducing PR-1 proteins.

Moreover, chitinase degrades the fungal pathogen cell wall by hydrolyzing the $\beta-1,4$-glucosidic bonds between chitin $\mathrm{N}$-acetylglucosamine monomers to further inhibit the infection (Okongo et al. 2019). It has been reported that chitinase can improve resistance to ear rot fungi in corn and resistance to the red rot pathogen (Colletotrichum falcatum Went) in barley and can inhibit fungal spore germination and mycelium growth (Dowd et al. 2018). In this study, we found that expression of 10 chitinase-encoding genes showed an up-regulation trend over the course of infection, indicating that chitinase accumulates in the pear to cope with A. alternata infection.

Thaumatin-like proteins (TLPs) are widely distributed in many organisms such as plants, fungi and insects (Meng et al. 2017). Previous studies have shown that TLPs have significant antifungal activity (Misra et al. 2016) and mainly work in two ways: (1) TLPs are directly inserted into the 
fungal plasma membrane to form perforations, thereby destroying membrane permeability; (2) TLPs can cause enzymatic hydrolysis of $\beta$-1,3-glucan, a major part of the fungal cell wall. We identified 10 TLP-encoding genes, of which seven were up-regulated following A. alternata infection, with TLP1 (LOC103935639) and TLPIa (LOC103962570, LOC103967809) showing significant increases in mRNA levels. These results indicate that $A$. alternata infection causes the up-regulated expression of genes encoding TLPs to resist pathogen infection in pear. Furthermore, heat shock proteins (HSPs), which act as molecular chaperones, can repair and remove the misfolded proteins produced by external factors such as plant stress, thereby reconstructing cellular protein homeostasis (Wang et al. 2004). Previous studies have shown that Arabidopsis infection by four pathogenic bacteria also caused the accumulation of HSP83, HSP70, HSP23.6, HSP17.6 A, and HSP17.4 (Whitham et al. 2003). In our experimental results, the genes encoding HSPs showed a down-regulated pattern of expression throughout the infection phase, which is consistent with results showing the down-regulation of genes encoding Hsfs transcription factors. It shows that $A$. alternate, in the process of infecting pear fruits, inhibits the expression of related transcription factors, thereby disrupting normal protein homeostasis and inhibiting the expression of defense responses. Down-regulation of the genes encoding HSP70 and HSP83 may be one of the important pathogenic mechanisms during the infection of Fragrant Pear fruits by A. alternata. Based on these results, we were able to draw a feasible molecular network that can explain the defense response in pear to A. alternata infection (Fig. 10). First of all, when pears are inoculated with $A$. alternata, the pathogen will destroy the integrity of the cell wall and a series of defense responses such as plant hormone signaling pathways (SA and JA, for example) and plant-pathogen interactions (PTI, ETI) are activated. Subsequently, TFs (WRKY, MYB, ERF, Hsfs) trigger the host responses to A. alternata infection by activating or inhibiting the expression of downstream genes including CHT, HSP and PR-1. Briefly, pears undergo specific changes in defense-related gene expression through molecular networks after infection with A. alternata and then produce related defense response.

\section{Conclusion}

In conclusion, in this study, a total of 25,877 DEGs were detected, and results showed that the DEGs take part in plant pathogen interactions and plant hormone signaling pathways, and defense-related proteins were up-regulated, suggesting a positive role for these genes in the pear- $A$. alternata interaction. Moreover, expression of the DEGs involved in cutin and wax biosynthesis was down-regulated, as are DEGs that encode TFs (WRKY, MYB, ERF, Hsfs) and HSP70 and HSP83, following infection of pear fruits by A. alternata, which may result in the appearance of blackhead disease symptoms. This study explored the defense mechanism and pathogenesis of pears in response to A. alternata infection and our results are expected to provide support for the development of new storage and preservation technologies for pears.

\section{Acknowledgements}

We acknowledge the financial supports of the National Natural Science Foundation of China under Grant No. 32060561; Research project of young and middle-aged leading scientists, engineers and innovators in Xinjiang production and construction corps (2018CB024); Scientific and technological research plan in key areas of the Corps, Research on the key technology of cold chain logistics and deep processing and creation of new products (2019AB024).

\section{Author Contributions}

Hui Ouyang and Guogang Chen conceived and designed the experiments; Hui Ouyang and Tongrui Sun performed the experiments; Hui Ouyang, Tongrui Sun, Minrui Guo, Weida Zhang analyzed the data; Ying Jiang contributed reagents; Fund acquisition from Shaobo Cheng and Guogang Chen.

\section{Conflict of Interest}

There are no conflicts to declare.

\section{Data Availability}

The data presented in this study are available on request from the corresponding author.

\section{Ethics Approval}

There are no researches conducted on animals or humans.

\section{References}

Aarts MG, CJ Keijzer, WJ Stiekema, A Pereira (1995). Molecular characterization of the CER1 gene of arabidopsis involved in epicuticular wax biosynthesis and pollen fertility. Plant Cell 7:2115-2127

Axtell MJ, BJ Staskawicz (2003). Initiation of RPS2-specified disease resistance in Arabidopsis is coupled to the AvrRpt2-directed elimination of RIN4. Cell 112:369-377

Bari R, JD Jones (2009). Role of plant hormones in plant defence responses. Plant Mol Biol 69:473-488

Bellincampi D, F Cervone, V Lionetti (2014). Plant cell wall dynamics and wall-related susceptibility in plant-pathogen interactions. Front Plant Sci 5; Article 228

Bethke G, RE Grundman, S Sreekanta, W Truman, F Katagiri, J Glazebrook (2014). Arabidopsis pectin methylesterases contribute to immunity against Pseudomonas syringae. Plant Physiol 164:1093-1107

Bhattacharjee S, MK Halane, SH Kim, W Gassmann (2011). Pathogen effectors target Arabidopsis EDS1 and alter its interactions with immune regulators. Science 334:1405-1408 
Cao FY, TA DeFalco, W Moeder, B Li, Y Gong, X M Liu, M Taniguchi, S Lumba, S Toh, L Shan, B Ellis, D Desveaux, K Yoshioka (2018). Arabidopsis ethylene response factor 8 (ERF8) has dual functions in ABA signaling and immunity. BMC Plant Biol 18; Article 211

Chen J, J Lu, Z He, F Zhang, S Zhang, H Zhang (2020). Investigations into the production of volatile compounds in Korla fragrant pears (Pyrus sinkiangensis Yu). Food Chem 302:325-337

Chen XF, LP Teng, HX Dan, RC Xiong (2019). Occurrence of black leaf spot caused by Alternaria alternata on Korla fragrant pear in Xinjiang of China. J Plant Pathol 102:265-265

Cheng SB, Y Jiang, MR Guo, LJ Nan, N Chen, YS Li, CW Cui, GG Chen (2019). Parsing of compositions and micro-structural characteristics for rust-spots of pear pericarp. Nanosci Nanotechnol Lett 11:441-450

Colcombet J, H Hirt (2008). Arabidopsis MAPKs: A complex signalling network involved in multiple biological processes. Biochem $J$ 413:217-226

Crabill E, A Joe, A Block, JMV Rooyen, JR Alfano (2010). Plant immunity directly or indirectly restricts the injection of type III effectors by the Pseudomonas syringae type III secretion system. Plant Physiol 154:233-244

Dalcero A, M Combina, M Etcheverry, S Chulze, MI Rodriquez (1996). Effect of dichlorvos on growth and mycotoxin production by Alternaria alternata. Food Addit Contam 13:315-320

Dezar CA, JI Giacomelli, PA Manavella, DA Ré, M Alves-Ferreira, IT Baldwin, G Bonaventure, RL Chan (2011). HAHB10, a sunflower HD-Zip II transcription factor, participates in the induction of flowering and in the control of phytohormone-mediated responses to biotic stress. J Exp Bot 62:1061-1076

Dowd PF, TA Naumann, NPJ Price, ET Johnson (2018). Identification of a maize (Zea mays) chitinase allele sequence suitable for a role in ear rot fungal resistance. Agric Gene 7:15-22

Gidda SK, JM Shockey, SJ Rothstein, JM Dyer, RT Mullen (2009). Arabidopsis thaliana GPAT8 and GPAT9 are localized to the ER and possess distinct ER retrieval signals: Functional divergence of the dilysine ER retrieval motif in plant cells. Plant Physiol Biochem 47:867-879

Guo M, F Tian, Y Wamboldt, JR Alfano (2009). The majority of the type III effector inventory of Pseudomonas syringae pv. tomato DC3000 can suppress plant immunity. Mol Plant Microb Interact 22:1069-1080

Jwa NS, GK Agrawal, SU Tamogami, M Yonekura, O Han, H Iwahashi, R Rakwal (2006). Role of defense/stress-related marker genes, proteins and secondary metabolites in defining rice self-defense mechanisms. Plant Physiol Biochem 44:261-273

Kan J, T Liu, N Ma, H Li, X Li, J Wang, B Zhang, Y Chang, J Lin (2017). Transcriptome analysis of Callery pear (Pyrus calleryana) reveals a comprehensive signalling network in response to Alternaria alternata. PLoS One 12; Article e0184988

Kumar M, W Busch, H Birke, B Kemmerling, T Nürnberger (2009). Heat shock factors HsfB1 and HsfB2b are involved in the regulation of Pdf1.2 expression and pathogen resistance in Arabidopsis. Mol Plant 2:152-165

Kurdyukov S, A Faust, S Trenkamp, S Bär, R Franke, N Efremova, K Tietjen, L Schreiber, H Saedler, A Yephremov (2006). Genetic and biochemical evidence for involvement of HOTHEAD in the biosynthesis of long-chain $\alpha$-, $\omega$-dicarboxylic fatty acids and formation of extracellular matrix. Planta 224:315-329

Laurens L,P Jacob, G Alain, B Rudi, S Jens (2017). Abscisic acid as pathogen effector and immune regulator Front Plant Sci 8; Article 587

Loon LCV, AV Kammen (1970) Polyacrylamide disc electrophoresis of the soluble leaf proteins from Nicotiana tabacum var. "Samsun" and "Samsun NN". II. Changes in protein constitution after infection with tobacco mosaic virus. Virology 40:190-211

Li F, X Wu, P Lam, D Bird, H Zheng, L Samuels, R Jetter, L Kunst (2008). Identification of the wax ester synthase/acyl-coenzyme A Diacylglycerol acyltransferase WSD1 required for stem wax ester biosynthesis in Arabidopsis. Plant Physiol 148:97-107

Ma L, L Zhou, S Quan, H Xu, J Yang, J Niu (2019). Integrated analysis of mRNA-seq and miRNA-seq in calyx abscission zone of Korla fragrant pear involved in calyx persistence. BMC Plant Biol 19; Article 192
Ma ZH, TJ Michailides (2005). Advances in understanding molecular mechanisms of fungicide resistance and molecular detection of resistant genotypes in phytopathogenic fungi. Crop Prot 24:853-863

Marguerat S, J Bähler (2010). RNA-seq: From technology to biology. Cell Mol Life Sci 67:569-579

Meng FL, J Wang, X Wang, YX Li, X Yao Zhang (2017). Expression analysis of thaumatin-like proteins from Bursaphelenchus xylophilus and Pinus massoniana. Physiol Mol Plant Pathol 100:178-184

Misra RC, M Kamthan, S Kumar, S Ghosh (2016). A thaumatin-like protein of Ocimum basilicum confers tolerance to fungal pathogen and abiotic stress in transgenic Arabidopsis. Sci Rep 6; Article 25340

Niderman T, I Genetet, T Bruyère, R Gees, A Stintzi, M Legrand, B Fritig, E Mösinger (1995). Pathogenesis-related PR-1 proteins are antifungal. Isolation and characterization of three 14-kilodalton proteins of tomato and of a basic PR-1 of tobacco with inhibitory activity against Phytophthora infestans. Plant Physiol 108:17-27

Okongo RN, AK Puri, ZX Wang, S Singh, K Permaul (2019). Comparative biocontrol ability of chitinases from bacteria and recombinant chitinases from the thermophilic fungus Thermomyces lanuginosus. $J$ Biosci Bioeng 127:663-671

Pajerowska-Mukhtar KM, W Wang, Y Tada, N Oka, CL Tucker, JP Fonseca, X Dong (2012). The HSF-like transcription factor TBF1 is a major molecular switch for plant growth-to-defense transition. Curr Biol 22:103-112

Pandey SP, IE Somssich (2009). The role of WRKY transcription factors in plant immunity. Plant Physiol 150:1648-1655

Robert-Seilaniantz A, M Grant, JDG Jones (2011). Hormone crosstalk in plant disease and defense: More than just jasmonate-salicylate antagonism. Аnnu Rev Phytopathol 49:317-343

Rose JK, J Braam, SC Fry, K Nishitani (2002). The XTH family of enzymes involved in xyloglucan endotransglucosylation and endohydrolysis: Current perspectives and a new unifying nomenclature. Plant Cell Physiol 43:1421-1435

Su SH, SM Bush, N Zaman, K Stecker, MR Sussman, P Krysan (2013). Deletion of a tandem gene family in Arabidopsis: Increased MEKK2 abundance triggers autoimmunity when the MEKK1-MKK1/2-MPK4 signaling cascade is disrupted. Plant Cell 25:1895-1910

Sun YD, L Li, AP Macho, ZF Han, ZH Hu, C Zipfel, JM Zhou, JJ Chai (2013). Structural basis for flg22-induced activation of the Arabidopsis FLS2-BAK1 immune complex. Science 342:624-628

Tian HL, P Zhan, ZY Deng, HY Yan, XR Zhu (2014). Development of a flavour fingerprint by GC-MS and GC-O combined with chemometric methods for the quality control of Korla pear (Pyrus serotina Reld). Intl J Food Sci Technol 49:2546-2552

Tian X, L Zhang, S Feng, Z Zhao, X Wang, H Gao (2019). Transcriptome analysis of apple leaves in response to powdery mildew (Podosphaera leucotricha) infection. Intl J Mol Sci 20:2326-2344

Vidhyasekaran P (2016). Molecular manipulation of transcription factors, the master regulators of PAMP-triggered signaling systems. In: Switching on Plant Innate Immunity Signaling Systems, pp:255-358. Vidhyasekaran P (Ed.). Springer Nature, Cham, Switzerland

Wang W, B Vinocur, O Shoseyov, A Altman (2004). Role of plant heatshock proteins and molecular chaperones in the abiotic stress response. Trends Plant Sci 9:244-252

Whitham SA, S Quan, HS Chang, B Cooper, B Estes, T Zhu, X Wang, YM Hou et al. (2003). Diverse RNA viruses elicit the expression of common sets of genes in susceptible Arabidopsis thaliana plants. Plant J 33:271-283

Wu J, Z Wang, Z Shi, S Zhang, R Ming, S Zhu, MA Khan, S Tao, SS Korban, H Wang, NJ Chen, T Nishio, X Xu, L Cong, K Qi, X Huang, Y Wang, X Zhao, J Wu, C Deng, C Gou, W Zhou, H Yin, G Qin, Y Sha, Y Tao, H Chen, Y Yang, Y Song, D Zhan, J Wang, L Li, M Dai, C Gu, Y Wang, D Shi, X Wang, H Zhang, L Zeng, D Zheng, C Wang, M Chen, G Wang, L Xie, V Sovero, S Sha, W Huang, S Zhang, M Zhang, J Sun, L Xu, Y Li, X Liu, Q Li, J Shen, J Wang, RE Paull, JL Bennetzen, J Wang, S Zhang (2013). The genome of the pear (Pyrus bretschneideri Rehd.). Genome Res 23:396-408 
Ouyang et al. / Intl J Agric Biol, Vol 25, No 6, 2021

Ya X, SS Liu, YQ Liu, S Ling, CS Chen, JL Yao (2017). HOTHEAD-Like HTH1 is involved in anther cutin biosynthesis and is required for pollen fertility in rice. Plant Cell Physiol 58:1238-1248

Yang Z, L Tian, M Latoszek-Green, D Brown, K Wu (2005). Arabidopsis ERF4 is a transcriptional repressor capable of modulating ethylene and abscisic acid responses. Plant Mol Biol 58:585-596

Yu XY, Y Yao, YH Hong, PY Hou, CX Li, ZQ Xia, MT Geng, YH Chen (2019). Differential expression of the Hsf family in cassava under biotic and abiotic stresses. Genome 62:563-569
Zhang XY, JY Qiu, QL Hui, YY Xu, YZ He, LZ Peng, XZ Fu (2020). Systematic analysis of the basic/helix-loop-helix (bHLH) transcription factor family in pummelo (Citrus grandis) and identification of the key members involved in the response to iron deficiency. BMC Genomics 21; Article 233

Zhu LM, WC Ni, S Liu, BH Cai, H Xing, SH Wang (2017). Transcriptomics analysis of apple leaves in response to Alternaria alternata apple pathotype infection. Front Plant Sci 8; Article 22 